\title{
Análisis económico de la producción de berenjena (Solanum melongena L.) en dos zonas productoras del Caribe colombiano: Sabanas de Sucre y Valle del Sinú en Córdoba
}

\author{
Antonio María Martínez-Reina ${ }^{1}$ \\ Lilibet Tordecilla-Zumaqué ${ }^{2}$ \\ Liliana María Grandett-Martínez ${ }^{3}$ \\ María del Valle Rodríguez-Pinto ${ }^{4}$ \\ Carina Cecilia Cordero-Cordero ${ }^{5}$ \\ Alfonso Rafael Orozco-Guerrero ${ }^{6}$ \\ Gabriel Ernesto Silva-Acosta ${ }^{7}$ \\ Jorge Luis Romero-Ferrer ${ }^{8}$ \\ Ender Manuel Correa-Álvarez ${ }^{9}$
}

Fecha de recepción: 6 de febrero de 2019

Fecha de aceptación: 15 de abril de 2019

\section{Resumen}

Este estudio describe las características socioeconómicas y tecnológicas del sistema de producción de berenjena en las microrregiones del Valle del Sinú y Sabanas de Colombia. A través de la técnica de muestreo aleatorio simple se seleccionaron 62 agricultores. Los datos fueron recolectados utilizando una encuesta formal estructurada, previamente probada y analizada. Se encontró que el cultivo es sembrado por pequeños productores en un área promedio de 0,6 hectáreas. La edad promedio de los productores es de 53 años con más de 30 años de experiencia en el cultivo. Resulta menos costoso producir berenjena en el departamento de Sucre que en Córdoba, lo que se explica por la proporción en que participa la mano de obra en los costos de producción, debido a que es mayor para el departamento de Sucre con el $75 \%$ del total de los costos; por el contrario, en el departamento de Córdoba la mano de obra participa en un $63 \%$. Los ingresos netos son mayores en el caso del departamento de Córdoba por la diferencia de los rendimientos, los cuales son de $35 \mathrm{t} \mathrm{ha}^{-1}$, en tanto que para el

\footnotetext{
1 Ph. D. Corporación Colombiana de Investigación Agropecuaria-AGROSAVIA (Turipaná-Córdoba, Colombia). amartinezr@agrosavia.co. ORCID: 0000-0002-9312-842X.

2 M. Sc. Corporación Colombiana de Investigación Agropecuaria-AGROSAVIA (Turipaná-Córdoba, Colombia). Itordecilla@agrosavia.co. ORCID: 0000-0003-0263-6427.

3 M. Sc. Corporación Colombiana de Investigación Agropecuaria-AGROSAVIA (Turipaná-Córdoba, Colombia). lgrandett@agrosavia.co. ORCID: 0000-0002-9755-2017.

4 M. Sc. Corporación Colombiana de Investigación Agropecuaria-AGROSAVIA (Turipaná-Córdoba, Colombia). mdelvrodriguez@agrosavia.co. ORCID: 0000-0002-4820-8287.

5 M. Sc. Corporación Colombiana de Investigación Agropecuaria-AGROSAVIA (Motilonia-Cesar, Colombia). ccordero@agrosavia.co. ORCID: 0000-0003-3688-5835.

6 Corporación Colombiana de Investigación Agropecuaria-AGROSAVIA (Caribia-Magdalena, Colombia). arorozco@agrosavia.co. ORCID: 0000-0001-6297-8918.

Corporación Colombiana de Investigación Agropecuaria-AGROSAVIA (Caribia-Magdalena, Colombia). gesilva@agrosavia.co. ORCID: 0000-0002-6519-8973.

8. Ph. D. Corporación Colombiana de Investigación Agropecuaria-AGROSAVIA (Turipaná-Córdoba, Colombia). ajromero@agrosavia.co. ORCID: 0000-0002-7249-6549.

9 M. Sc. Corporación Colombiana de Investigación Agropecuaria-AGROSAVIA (Caribia-Magdalena, Colombia). ecorrea@agrosavia.co. ORCID: 0000-0001-7876-5052.
} 
departamento de Sucre son de $25 \mathrm{t} \mathrm{ha}^{-1}$. Con relación a los márgenes de comercialización, por cada unidad monetaria que paga el consumidor, $\$ 0,82$ constituyen utilidades que se distribuyen en la cadena de intermediación, la cual corresponde a un valor muy alto, siendo este de un $53 \%$. Se concluye que el agricultor es el que más arriesga y el que menos recibe de esta diferencia del precio entre el agricultor y el consumidor final.

Palabras clave: consumidor; costos de producción; indicadores económicos; márgenes de comercialización; mercados.

\section{Economic Analysis of the Production of Eggplant (Solanum melongena L.) in Two Producing Areas of the Colombian Caribbean: Sucre Savannas and Sinú Valley in Córdoba}

\section{Abstract}

This paper describes the socioeconomic and technological characteristics of the eggplant production system in the microregions of the Sinú Valley and Sucre Savannas in Colombia. Through the simple random sampling technique, we selected 62 farmers. It was collected data using a formal structured survey previously tested and analyzed. Small producers plant the crop in an average area of 0.6 hectares. The average age is 53 years with more than 30 years of experience in cultivation. It is less expensive to produce eggplant in the state of Sucre than in Córdoba, due to the proportion in which labor is involved in production costs, because they are higher for Sucre State with $75 \%$ of the total costs, on the contrary, in Córdoba State the labor force participates in $63 \%$. The net income is higher in the case of Córdoba owing to the difference in yields, which are $35 \mathrm{t} / \mathrm{ha}^{-1}$ while for Sucre they are $25 \mathrm{t} / \mathrm{ha}^{-1}$. With regard to marketing margins, for each monetary unit that the consumer pays, $\$ 0.82$ constitutes profits that are in the intermediation chain distributed. The participation of $53 \%$ intermediation is very high. We conclude that the farmer is the one who risks the most and who receives the least from this difference in the price between the farmer and the final consumer.

Keywords: consumer; economic indicators; marketing margins; markets; production costs.

\footnotetext{
Para citar este artículo:

Martínez-Reina, A. M., Tordecilla-Zumaqué, L., Grandett-Martínez, L. M., Rodríguez-Pinto, M. del V., Cordero-Cordero, C. C., Orozco-Guerrero, A. R., Silva-Acosta, G. E., RomeroFerrer, J. L., \& Correa-Álvarez, E. M. (2019). Análisis económico de la producción de berenjena (Solanum melongena L.) en dos zonas productoras del Caribe colombiano: Sabanas de Sucre y Valle del Sinú en Córdoba. Ciencia y Agricultura, 16(3), 17-34 https://doi.org/10.19053/01228420.v16.n3.2019.9514.
}

Esta obra está bajo licencia internacional Creative Commons Reconocimiento 4.0

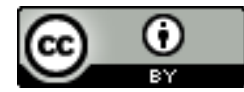

Ciencia y Agricultura (Cien. Agri.) Vol. 16 (3). L-ISSN 0122-8420. elSSN 2539-0899.

Septiembre-Diciembre 2019, pp. 17-34. Tunja (Boyacá) - Colombia. DOI: https://doi.org/10.19053/01228420.v16.n3.2019.9514 


\section{INTRODUCCIÓN}

La producción de hortalizas en la región Caribe no ha sido una actividad importante dentro del grupo de productos agrícolas; por eso, la oferta de productos hortícolas no es abundante y su cultivo se hace en áreas muy pequeñas; sin embargo, sobresalen algunas especies como ahuyama, berenjena, ají dulce y habichuela larga, de las cuales la berenjena ocupa un tercer lugar en área cultivada dentro del grupo de especies en esta región del país (Agronet, 2018a).

La berenjena es cultivada en seis departamentos, en un área de 253 ha y una producción de $2616 \mathrm{t}$, con rendimientos muy variables que oscilan entre 8 y 40 t/ha ${ }^{-1}$ dependiendo del nivel de tecnificación implementado. En el ámbito nacional, Córdoba es el cuarto productor con una participación del 10,33 \% de la producción de berenjena, después de Magdalena con un 53,99\%, Bolívar con el $18,45 \%$ y Valle del Cauca con el 13,20 \% (Agronet, 2018b). Esta especie es cultivada en áreas que oscilan entre 1000 y $5000 \mathrm{~m}^{2}$, manejadas con deficiente tecnología y serios problemas de comercialización (Araméndiz, Cardona, Jarma \& Espitia, 2008).

Pese a la gran importancia que representa el cultivo de berenjena en la región Caribe, es poca la disponibilidad de información en cuanto a los aspectos tecnológicos, socioeconómicos y de mercados, los cuales son de gran interés cuando se quieren planificar actividades de investigación o de fomento en la producción de hortalizas, por lo cual se corre el riesgo de formular opciones tecnológicas y políticas equivocadas.

Diversas metodologías se han desarrollado en el tema de la caracterización de sistemas de producción agrícola (Agreda, Quijandría \& Ruiz, 1991; Escobar \& Berdegué, 1990), varios autores han llevado a cabo estudios de caracterización de los sistemas de producción de maíz (Espinosa, Játiva \& Suárez, 1990), lulo (Ríos et al., 2004), papa (Vallejos, 2012), y cacao (Pabón, 2016). Por su parte, Martínez (2013) determinó los sistemas de producción existentes en la región de La Mojana sucreña (Colombia). En hortalizas, los trabajos más recientes se han dedicado al frijol (Torres et al., 2013) y tomate (Alam, Uddin, Moniruzzaman, Nazia \& Haque, 2016).

Con relación a los márgenes de comercialización, Martínez y Esquivia (2007) hicieron un estudio en el que se calculó la participación directa del productor en el sistema de producción de yuca seca dentro de la cadena avícola. Dicho estudio mostró que se generó un margen bruto de $27 \%$ y una participación directa del productor del $73 \%$, lo cual indica la baja participación de los agricultores en los beneficios generados a lo largo de la cadena producción-consumo.

Posteriormente se han hecho estudios con enfoque de cadena productiva, como el de Arvizu, Mayett, Martínez, Olivares y Flórez (2015), quienes analizaron la producción de hortalizas desde el punto de vista de la cadena de valor obtenida 
con información directa de los agentes que participan en la producción y comercialización de hortalizas en cuatro municipios del Distrito de Desarrollo Rural de Tecamachalco, Puebla, México. La metodología usada fue la metodología funcional, de cadena de valor y de LEADER. Concluye el estudio que los beneficios esperados por los agricultores no son buenos, debido principalmente a la alta intermediación y la baja integración de la cadena, que hacen que el agricultor tenga una participación muy baja en los márgenes de comercialización.

La información sobre retornos económicos y distribución de los márgenes de comercialización a través de los diferentes actores que intervienen en la cadena producción-consumo fue generada en el estudio de línea base y es la que constituye el insumo principal para el presente artículo. El objetivo de la investigación fue realizar un análisis de los retornos económicos y los márgenes de comercialización en el sistema de producción de la berenjena, con el fin de entender la transferencia del valor y la distribución de utilidades en los distintos actores que intervienen desde la producción en finca hasta el consumidor final.

La hipótesis de la cual parte esta investigación es que la producción de hortalizas en la región Caribe, y particularmente la berenjena, no está debidamente documentada en las variables tecnológicas y socioeconómicas, y por esta razón se desconoce la información sobre los retornos a la inversión y la participación del productor en los beneficios de la producción a lo largo de la cadena producciónconsumo.

\section{MATERIALES Y MÉTODOS}

El estudio se llevó a cabo en las zonas productoras de berenjena de dos microrregiones del Caribe colombiano: el Valle del Sinú, que para este estudio comprende los municipios de San Pelayo, Cereté, Montería y San Bernardo del Viento, en el departamento de Córdoba, y las Sabanas que comprenden los municipios de Sincelejo, Corozal, San Antonio de Palmito y Sampués, en el departamento de Sucre.

La técnica de muestreo fue el estratificado simple para muestras pequeñas (Rodríguez, 2005), que partió de un universo de 846 agricultores, datos obtenidos de las evaluaciones agropecuarias y de Agronet (2018b). Se tomó como variable de muestreo el tamaño de la unidad productiva, lo cual especifica que hay unidades productivas de menos de una hectárea cultivada que corresponden al 62 $\%$ de la población. El otro componente son los agricultores que siembran más de una hectárea y constituyen el $38 \%$ del universo.

Para el cálculo del tamaño de la muestra se usó el muestreo aleatorio simple para muestras pequeñas, de acuerdo con Rodríguez (2005). Para el cálculo del tamaño de la muestra se aplicó la siguiente fórmula: 


$$
n=\frac{\mathrm{Z}^{*} p^{*} q^{*} N}{\left[\left(e^{2} *(N-1)\right)+\mathrm{Z}^{2} * p^{*} q\right]}
$$

Donde

$z_{\alpha / 2}: z$ correspondiente al nivel de confianza elegido, en este caso el $95 \%$.

p: proporción de la población que cultiva menos de una hectárea: 524 agricultores.

q: la diferencia entre el total de la población y la proporción de la población que no reúne la característica (en este caso unidades mayores de una hectárea): 321 agricultores.

e: error máximo $14,7 \%$.

$\mathrm{N}$ : tamaño de la población: 846 unidades productivas.

La muestra fue de 62 agricultores a los cuales se les aplicó la encuesta.

Para la recolección de la información se usó una encuesta formal estructurada, compuesta por 28 variables, en dos bloques de preguntas: unas de tipo técnico y otras de tipo socioeconómico. La información obtenida fue tabulada y procesada en una base de datos en el programa SPSS (IBM SPSS) por medio de análisis estadísticos descriptivos de frecuencia, tablas de contingencia y análisis de correlación.

La información sobre costos de producción, indicadores económicos y márgenes de comercialización del cultivo se obtuvo mediante el desarrollo de dos talleres donde participaron agricultores de amplia trayectoria en el cultivo de berenjena provenientes del Valle del Sinú (Córdoba) y Sabanas de Sucre. En cada taller se aplicó el método del consenso, siguiendo el esquema metodológico participativo descrito en la Figura 1. Los parámetros de costos (directos, indirectos y totales), rentabilidad, valores unitarios, punto de equilibrio, etc., fueron determinados con base en la teoría económica de Krugman y Wells (2006) (Tabla 1).

La información tecnológica del manejo del cultivo se interpretó al lenguaje económico mediante los patrones de costos de producción, para lo cual se llevaron a cabo dos talleres: uno en el Valle del Sinú (C.I. Turipaná, Cereté, Córdoba) y otro en la Sabana (Sincelejo, Sucre). Con la información obtenida se calcularon retornos económicos a través de la identificación de los elementos constitutivos del costo total de producción, como mano de obra e insumos.

Con los rendimientos se calcularon los costos unitarios y los retornos económicos, como ingresos netos, rentabilidad, punto de equilibrio y eficiencia. La mano de obra se calculó de acuerdo con el valor del jornal en la región, y el precio de los insumos, de acuerdo con CIMMYT (1988), es decir, el precio que paga el agricultor por el producto más el costo del transporte por llevarlo a la finca. 
Análisis económico de la producción de berenjena (Solanum melongena L.) en dos zonas productoras del Caribe colombiano: Sabanas de Sucre y Valle del Sinú en Córdoba

1. Listado de actividades y definición de la tecnología que utiliza el productor.
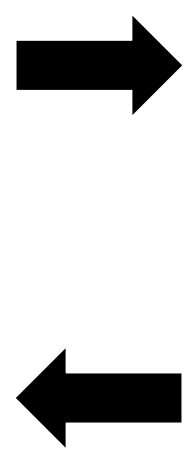

2. Definición de unidades para tener en cuenta en el proceso de construcción de patrones de costos.
4. Ponderación de los diferentes
componentes del sistema de
producción.

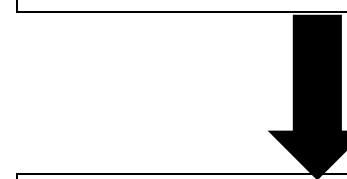

5. Elaboración del documento final con su respectivo análisis.

Fig. 1. Esquema metodológico participativo para la estimación de patrones de costos e indicadores económicos en sistemas productivos agropecuarios (Agreda et al., 1991).

Tabla 1. Parámetros de costos de producción, indicadores económicos y márgenes de comercialización estimados para el cultivo de berenjena (Solanum melongena L.) en zonas productoras del Valle del Sinú (Córdoba) y Sabanas de Sucre.

\begin{tabular}{|c|c|}
\hline Ecuación & Donde \\
\hline$C d=C d 1+C d 2+C d 3+\cdots+C d n$ & $\begin{array}{lccc}\text { Cd: costos directos; } \mathrm{Cd}_{1}: & \text { semillas; } & \mathrm{Cd}_{2}: \\
\text { agroquímicos; } \mathrm{Cd}_{3}: \text { jornales, etc. } & \end{array}$ \\
\hline$C l$ & $\begin{array}{l}\text { Ci: costos indirectos; } \mathrm{Ci}_{1} \text { : alquiler del terreno; } \mathrm{Ci}_{2} \text { : } \\
\text { costos financieros; } \mathrm{Ci}_{3}: \text { otros, etc. }\end{array}$ \\
\hline$C P=C d+C i$ & $\begin{array}{l}\text { CP: costos de producción; Cd: costos directos; } \mathrm{Ci} \text { : } \\
\text { costos indirectos. }\end{array}$ \\
\hline$\sum_{R d o=0} R d o=P C 1+P C$ & $\begin{array}{l}\text { Rdo: rendimiento; } \mathrm{PC}_{1} \text { : producción primera } \\
\text { cosecha; } \mathrm{PC}_{2} \text { : producción segunda cosecha; } \mathrm{PC}_{3} \text { : } \\
\text { producción tercera cosecha; } \mathrm{PC} \text { : producción } \\
\text { última cosecha. }\end{array}$ \\
\hline$C U=\frac{C P}{R d a}$ & $\begin{array}{l}\text { CU: costos unitarios; CP: costos de producción; } \\
\text { Rdo: rendimiento. }\end{array}$ \\
\hline$I B=R$ do* $P V$ & $\begin{array}{l}\text { IB: ingresos brutos; Rdo: rendimiento; PV: precio } \\
\text { de venta de la producción de berenjena. }\end{array}$ \\
\hline$I N=I B-C P$ & $\begin{array}{l}\text { IN: ingresos netos; IB: ingresos brutos; CP: costos } \\
\text { de producción. }\end{array}$ \\
\hline$R T=\frac{I B-C d}{C d} * 100$ & $\begin{array}{l}\text { RT: rentabilidad técnica; IB: ingreso bruto; Cd: } \\
\text { costos directos. }\end{array}$ \\
\hline$R N=\frac{I B-C P}{C P} * 100$ & $\begin{array}{l}\text { RN: rentabilidad neta; IB: ingreso bruto; Cd: costos } \\
\text { de producción. }\end{array}$ \\
\hline$P e q=\frac{C P}{P V}$ & $\begin{array}{l}\text { Peq: punto de equilibrio; Cd: costos de producción; } \\
\text { PV: precio de venta. }\end{array}$ \\
\hline$P P e q=\frac{C P}{J B} * 100$ & $\begin{array}{l}\text { PPeq: porcentaje del punto de equilibrio; Cd: } \\
\text { costos de producción; IB: ingresos brutos. }\end{array}$ \\
\hline
\end{tabular}

Ciencia y Agricultura (Cien. Agri.) Vol. 16 (3). L-ISSN 0122-8420. elSSN 2539-0899.

Septiembre-Diciembre 2019, pp. 17-34. Tunja (Boyacá) - Colombia. DOI: 
Antonio María Martínez-Reina; Lilibet Tordecilla-Zumaqué; Liliana María Grandett-Martínez; María del Valle Rodríguez-Pinto; Carina Cecilia Cordero-Cordero; Alfonso Rafael Orozco-Guerrero; Gabriel Ernesto SilvaAcosta; Jorge Luis Romero-Ferrer; Ender Manuel Correa-Álvarez

\begin{tabular}{|c|l|}
\hline Ecuación & \multicolumn{1}{c|}{ Donde } \\
\hline$E t=\frac{P V}{C U} * 100$ & $\begin{array}{l}\text { Et: eficiencia técnica; PV: precio de venta; CU: } \\
\text { costos unitarios. }\end{array}$ \\
\hline$M B C=\frac{P C-P p}{P C} * 100$ & $\begin{array}{l}\text { MBC: margen bruto de comercialización; Pc: precio } \\
\text { del consumidor; Pp: precio del productor. }\end{array}$ \\
\hline$P D P=100-M B C$ & $\begin{array}{l}\text { PDP: participación directa del productor; MBC: } \\
\text { margen bruto de comercialización. }\end{array}$ \\
\hline$M N C=\frac{M B C-C m}{P C} * 100$ & $\begin{array}{l}\text { MNC: margen neto de comercialización; MBC: } \\
\text { margen bruto de comercialización; Cm: costos de } \\
\text { mercadeo; Pc: precio del consumidor. }\end{array}$ \\
\hline
\end{tabular}

Para el caso de las labores agronómicas en campo se aplicó la medida de tendencia central moda, considerando aquellas actividades que realizan los agricultores en el sistema de producción de berenjena en la región de acuerdo con Agreda et al. (1991). Para el cálculo del precio del producto cosechado se tuvo en cuenta el valor que le pagan al productor los intermediarios de la región, este valor se multiplicó con el rendimiento promedio calculado a partir de la información aportada por las encuestas y los talleres y se obtuvo el ingreso bruto, que al restarle los costos de producción permitió determinar el ingreso neto y la rentabilidad (definida como la relación entre el ingreso neto y el costo total). Además, se estimaron las cantidades mínimas de producción requeridas por parte de los agricultores para nivelar los ingresos con los costos, lo cual es conocido como el punto de equilibrio.

La eficiencia del sistema de producción se calculó siguiendo a Forero et al. (2013) quienes proponen los indicadores de rentabilidad técnica del cultivo, rentabilidad neta, eficiencia técnica y eficiencia económica. La rentabilidad técnica se determinó estableciendo la relación entre la diferencia en el ingreso bruto y los costos directos; para la rentabilidad neta se relacionó la diferencia entre el ingreso bruto y los costos directos e indirectos con el total de los costos, es el mismo concepto de rentabilidad que se usa tradicionalmente. Para calcular la eficiencia técnica del cultivo, se relacionó la diferencia entre el ingreso bruto y el costo directo con el área cultivada, es una manera de ver el aporte por área a la rentabilidad.

La intervención de diferentes actores durante el proceso de producción y comercialización de los productos origina variaciones en los precios en la medida que avanza de un eslabón de la producción a otro, es decir, el precio que le pagan al productor en finca, al intermediario y, por último, el que paga el consumidor final; se establece así el concepto de margen bruto de comercialización (MBC). Así mismo, se estimó la participación directa del productor (PDP) y el margen neto de comercialización (MNC) siguiendo los cálculos sugeridos por Espinosa, Rivera y García (2008) (Tabla 1). 


\section{RESULTADOS Y DISCUSIÓN}

Los agricultores de berenjena de los departamentos de Córdoba y Sucre se destacan por estar en una edad promedio de 53 años, con una amplia experiencia en el cultivo de 30 años; el $18 \%$ de los encuestados no presenta ningún nivel de estudio, mientras que el $39 \%$ tiene estudios hasta el nivel de educación básica primaria, un $25 \%$ estudió el bachillerato y solo un $11 \%$ alcanzó educación superior universitaria, el $5 \%$ del total de agricultores inició estudios universitarios pero no logró alcanzar el título, y un solo productor reporta estudios de posgrado, que representa el $2 \%$ de la muestra.

En relación con el área de siembra, el $62 \%$ de los agricultores establece su cultivo en áreas promedio de 0,6 hectáreas, donde el área máxima de siembra es de tres hectáreas; el $62 \%$ de los agricultores es propietario, de este porcentaje, el $33 \%$ adquirió la tierra por compra y el $29 \%$ por herencia o propiedad familiar. En cuanto a las labores del cultivo, predomina el uso de mano de obra contratada en un $73 \%$, destinada a labores de siembra y recolección principalmente, un $27 \%$ es mano de obra familiar, la cual es empleada para todas las labores requeridas en el cultivo. El $79 \%$ de los agricultores entrevistados usa semilla no certificada. Un $95 \%$ de la producción tiene como destino el mercado local, y un $5 \%$, el consumo de las familias.

La tecnología local de producción para el cultivo de la berenjena, según el trabajo de campo, es la siguiente: la fecha de siembra se establece de acuerdo con el inicio de las lluvias, dado que está condicionada a las precipitaciones y la disponibilidad de agua. En la selección del lote tienen en cuenta diferentes elementos de riesgo, como susceptibilidad a inundación, cercanía a una fuente de agua, proliferación de enfermedades radiculares a causa de la no rotación. Los suelos donde se cultiva la berenjena son arcillosos, de color oscuro, ricos en materia orgánica y bien drenada, profundos para facilitar el desarrollo radicular y el drenaje interno. Los preparan mecánica y manualmente. Realizan labores culturales de mantenimiento, como podas de formación, control manual de malezas, aporque aclareo de frutos, recolección de frutos dañados, las cuales se llevan a cabo durante todo el ciclo productivo del cultivo. La selección de la semilla es una de las labores previas a la siembra, los productores de berenjena utilizan distintos genotipos en sus cultivos. La totalidad de estos son variedades locales y corresponden a genotipos que han sido seleccionados en la zona productora 0 han sido traídos de otras localidades. Se destaca la adopción de variedades certificadas, tales como la C015 y C029, las cuales han sido liberadas por Agrosavia C.I. Turipaná como genotipos de alto rendimiento y con aceptación en el mercado local e internacional. Generalmente usan como criterio de selección los rendimientos y aceptabilidad en el mercado.

El sistema de producción que predomina es el monocultivo, que lo hace el $100 \%$ de la muestra encuestada. La producción del material de siembra tiene lugar en la misma finca, cada agricultor hace su propio semillero en forma tradicional, que consiste en un almácigo donde siembra muchas semillas extraídas de las 
berenjenas que él mismo cosecha en el cultivo anterior. En contadas excepciones compran la semilla. En este tipo de almácigos, la semilla es vertida directamente sobre la cama de siembra, la cual ha sido previamente desinfectada con agua caliente, formol o una solución fungicida. La adición de material orgánico al sustrato varía conforme la disponibilidad de restos vegetales de fácil descomposición.

Los agricultores siembran entre los meses de abril-junio, fecha que coincide con la primera época de lluvias del año. Un porcentaje más bajo de agricultores siembra entre los meses octubre-diciembre, y algunos que disponen de infraestructura de riego lo hacen entre los meses enero-marzo y julio- septiembre. En algunos casos también realizan resiembras 15 días después del trasplante, cuando el porcentaje de mortalidad no supera el $10 \%$. Usan plantas que fueron reservadas para tal fin y producidas en el mismo semillero.

Más de un $70 \%$ de los agricultores fertiliza químicamente, en tanto que un porcentaje bajo usa fertilizantes biológicos. La primera aplicación tiene lugar a los 21 días después de emergido (DDE); la segunda aplicación, a los 38 DDE. Una proporción muy baja de los agricultores hace la tercera aplicación a los 57 y 73 DDE, respectivamente. En general, los productores aplican en forma recurrente los fertilizantes urea y Triple 15 (N-P-K), en las dos primeras fertilizaciones, puesto que a medida que avanza el ciclo productivo del cultivo, la demanda nutricional es mayor y los agricultores aumentan las dosis de los fertilizantes. Únicamente el $3 \%$ de los agricultores realiza la fertilización con elementos menores, debido a que el mecanismo de aplicación es a través de productos de absorción foliar. Y solo el $6 \%$ de los agricultores emplea la enmienda de abonos orgánicos (Lombriabono) como fuente suplementaria de la nutrición de los cultivos.

Con relación al control de arvenses, un porcentaje lo hace químico y otro porcentaje lo hace combinando métodos de manual y mixto (manual + químico), que incluye la labor de limpia manual y aplicación de herbicidas. Un porcentaje bajo de los agricultores emplea elementos mecánicos para el control de malezas, mientras que un grupo más pequeño hace el control de malezas totalmente con herbicidas. Cabe destacar que los agricultores no identifican las malezas que se presentan en sus lotes y tampoco emplean coberturas vegetales, lo cual refleja que no realizan un manejo integrado de arvenses en el cultivo de berenjena. Al respecto, Hernández, Cardona, Vergara, Araméndiz y Velásquez-Arteaga (2015) señalan que las coberturas plásticas y orgánicas resultaron ser el mejor método de control de arvenses empleado en el cultivo de berenjena, al evaluar diferentes alternativas de manejo combinadas con y sin la presencia de micorrizas en el suelo; además, estos autores anotan que se demostró que el uso de glifosato influyó de manera negativa tanto en la colonización de las micorrizas como en el desarrollo de la planta.

Referente a las plagas y su manejo, las que causan mayor daño a los cultivos son la mosca blanca (Bemisia tabaci) y el ácaro rojo (Tetranychus ludeni), siendo estas 
las plagas de mayor incidencia en la época de verano; en tanto que el picudo (Faustinus spp) y el gusano comedor de follaje (Spodoptera spp) son plagas que pueden presentarse tanto en época de lluvia como en época de verano. Estos resultados coinciden con los reportados por Tapia et al. (2015), quienes mencionan este grupo de plagas, entre otros, como el de mayor interés en el cultivo, reportado por productores de berenjena en la región Caribe de Colombia.

Para el control de insectos plagas se utilizan insumos de síntesis química en un 39 $\%$ de la muestra encuestada, el $3 \%$ hace control bilógico, un $8 \%$ combina química con biológico, mientras que el $50 \%$ no hace ningún tipo de aplicaciones. Los productos de síntesis química empleados para el control insectos plagas, en su mayoría, son de amplio espectro, altamente residuales y contaminantes de las fuentes de agua. Los insecticidas más utilizados son a base de Cipermetrina, Dimetoato (Roxión) y Piryproxyfen (Epingle). A pesar de la larga lista de productos que se utilizan en el cultivo, existen diversas falencias en cuanto a medidas de protección por parte del agricultor, las formas de uso, rotación de moléculas y disposición final de los envases.

En lo relativo a otros problemas fitosanitarios en el cultivo de berenjena, los productores asocian la sintomatología patológica de enfermedades de origen fungoso a la marchitez causada por Fusarium spp y Sclerotium spp., respectivamente, las cuales suelen aparecer en la etapa productiva del cultivo.

Las enfermedades que más reportaron los agricultores fueron: antracnosis, fusarium, quemazón, mancha de las hojas y pudrición del fruto. Con relación al control, el $58 \%$ de la muestra encuestada afirma no utilizar ningún método de control, el $3 \%$ hace control bilógico, el $35 \%$, control químico, y un $4 \%$ emplea otros métodos de control, como eliminación de material enfermo, hojas secas.

La cosecha o recolección del fruto se efectúa de manera manual; se calcula que, en promedio, se realizan entre 21 a 40 cosechas en cultivos con un ciclo productivo de 270 días. Los rendimientos promedio por hectárea son de 432 bultos tres rayas, que equivalen a $21,6 \mathrm{t} / \mathrm{ha}$, con unos ingresos de $\$ 11.232 .000$ por hectárea y un ingreso neto de $\$ 6.477 .400$ por hectárea. La venta del producido se lleva a cabo preferiblemente en el lote. Algunos productores hacen acuerdos comerciales con los intermediarios basados en la fidelidad de compra en las fechas de mayor oferta; en todo caso, estos acuerdos son informales y verbales.

Con respecto a la evaluación económica del sistema de producción de berenjena en las dos localidades en estudio, se encontró que las labores se inician con la arada y rastrillada, ahoyada, elaboración del semillero, siembra de la plántula, control de malezas, plagas y cosecha, tutorado, amarre, recolección del producto, empaque y venta.

La información sobre los costos de una hectárea de berenjena en las dos localidades de Córdoba y Sucre permite hacer un análisis comparativo de los distintos elementos que intervienen en la producción, los cuales se presentan en la 
Tabla 2, en donde se observa que los ingresos netos son mayores en el caso del departamento de Córdoba. Lo cual se explica por la diferencia de los rendimientos que son de $35 \mathrm{t} / \mathrm{ha}^{-1}$, en tanto que para el departamento de Sucre son de $25 \mathrm{t} / \mathrm{ha}^{-1}$. Esto hace que la rentabilidad también sea mayor para el caso del departamento de Córdoba

La información obtenida en consenso del departamento de Sucre registra que los costos totales de producción de un cultivo de una hectárea de berenjena se calcularon en 5.002.470 pesos colombianos, de los cuales el $80 \%$ corresponde a los costos directos, y el $20 \%$ a los costos indirectos. A su vez, la mano de obra participa en el $75 \%$ del total de costos, y los insumos, en el $6 \%$ de los costos totales (Tabla 2).

Para el departamento de Córdoba, los costos totales de producción para una hectárea de un cultivo de berenjena se calcularon en $\$ 9.138 .420 \mathrm{ha}^{-1}$, de los cuales el $88 \%$ corresponde a los costos directos, y el $12 \%$ a los costos indirectos (Tabla 2). A su vez, la mano de obra participa en el $63 \%$ del total de costos, y los insumos en el $22 \%$ del total de costos (Figura 2).

El análisis de costos permite comparar los elementos constitutivos del costo en las dos microrregiones -Valle del Sinú y Sabanas- lo cual se aprecia en la Figura 2.

\section{Costos berenjena Sucre}

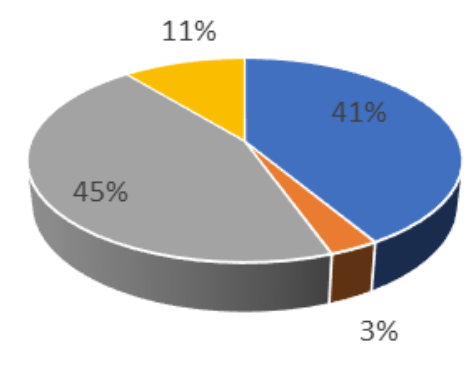

- Labores

- Costos Directos
- Insumos

- Costos Indirectos

\section{Costos berenjena Córdoba}

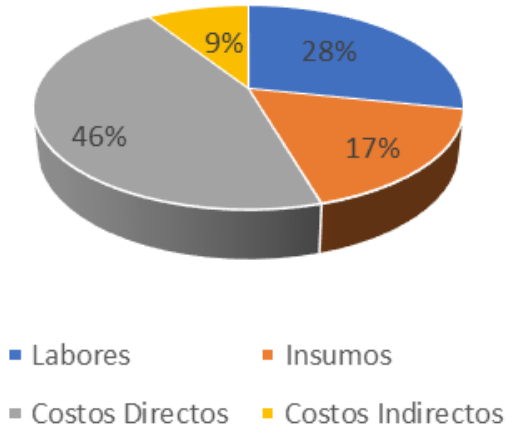

Fig. 2. Comparación de los elementos constitutivos del costo de producción en las microrregiones de los departamentos de Córdoba y Sucre.

Estas estructuras de costos tienen en común la participación de los costos directos, y difieren en la participación de las labores de manejo agronómico, siendo en mayor proporción para el caso del departamento de Sucre con un $41 \%$ de los costos totales, en tanto que para el departamento de Córdoba lo es en un $28 \%$. La participación de los insumos, aunque un poco lejana, guarda relación entre las dos localidades analizadas. Sin embargo, esto va a incidir en los análisis de los retornos a la inversión, los cuales se explican más adelante. 
Análisis económico de la producción de berenjena (Solanum melongena L.) en dos zonas productoras del Caribe colombiano: Sabanas de Sucre y Valle del Sinú en Córdoba

Tabla 2. Análisis comparativo de los costos de producción e indicadores económicos por hectárea para el cultivo de berenjena en zonas productoras del Valle del Sinú en Córdoba y Sabanas de Sucre, año 2018.

\begin{tabular}{|c|c|c|c|c|c|}
\hline \multirow{2}{*}{ Costos de producción } & \multicolumn{2}{|l|}{ Córdoba } & \multicolumn{2}{|l|}{ Sucre } & \multirow{2}{*}{$\begin{array}{c}\text { Diferencia } \\
\text { COP } \$\end{array}$} \\
\hline & COP \$ & $\mathbf{P}(\%)$ & COP \$ & $\mathbf{P}(\%)$ & \\
\hline Labores & 4.731 .000 & 51,8 & 3.740 .000 & 74,8 & 991.000 \\
\hline Insumos & 2.875 .000 & 31,5 & 281.000 & 5,6 & 2.594 .000 \\
\hline Costos directos & 7.606 .000 & 83,2 & 4.021 .000 & 80,4 & 3.585 .000 \\
\hline Costos indirectos & 1.532 .420 & 16,8 & 981.470 & 19,6 & 550.950 \\
\hline \multirow[t]{3}{*}{ Costos totales } & 9.138 .420 & & 5.002 .470 & & 4.135 .950 \\
\hline & \multicolumn{4}{|c|}{ Indicadores económicos } & \\
\hline & Córdoba & & Sucre & & \\
\hline Rendimiento (t/ha) & 35 & & 25 & & 10 \\
\hline Precio de venta $(\$ / t)$ & 400.000 & & 300.000 & & 100.000 \\
\hline Costo unitario $(\$ / t)$ & 261.098 & & 198.511 & & 62.587 \\
\hline Ingreso bruto (\$/ha) & 14.000 .000 & & 7.560 .000 & & 6.440 .000 \\
\hline Ingreso neto (\$/ha) & 4.861 .580 & & 2.557 .530 & & 2.304 .050 \\
\hline Rentabilidad técnica (\%) & 84,1 & & 88,0 & & $-3,9$ \\
\hline Rentabilidad neta (\%) & 53,2 & & 51,1 & & 2,1 \\
\hline Punto de equilibrio (t/ha) & 22,8 & & 16,7 & & 6,2 \\
\hline Punto de equilibrio (\%) & 65,3 & & 66,2 & & $-0,9$ \\
\hline Eficiencia & 1,5 & & 1,5 & & 0,0 \\
\hline
\end{tabular}

Nota: COP: pesos colombianos; P: participación.

Fuente: elaboración propia (datos, talleres participativos con productores).

La información de la Tabla 2 sobre los costos de producción permite apreciar que a pesar de ser el mismo sistema de producción, hay diferencias en las dos localidades, representadas en un valor de $4.135 .950 \$ / \mathrm{ha}^{-1}$. Esto se explica porque la proporción en que participa la mano de obra en los costos de producción es mayor en el caso del departamento de Sucre, con el $75 \%$ del total de los costos; lo que significa que es más intensivo en el uso de mano de obra. Por el contrario, en el departamento de Córdoba la mano de obra participa en un $63 \%$ y los insumos en un $26 \%$ y esto hace que el costo de producción sea más alto. Situación que puede estar relacionada con factores ambientales y de manejo agronómico, que influyen en una menor presión de plagas y enfermedades en comparación con el departamento de Córdoba, las cuales se reflejan en la cantidad de insumos empleados para controles fitosanitarios.

En cuanto a la eficiencia del sistema de producción, los resultados muestran un coeficiente de $88 \%$ para el caso de la producción del departamento de Sucre y un $84 \%$ para el departamento de Córdoba. Esta diferencia a favor del departamento de Sucre se explica por el bajo costo unitario, menor en $\$ 62.000$ con relación al departamento de Córdoba (Tabla 2). Lo que significa que técnicamente es más 
eficiente la producción en Sucre, pues, aunque son mejores las condiciones del departamento de Córdoba, son mejor aprovechados los recursos en el departamento de Sucre.

Con relación a la rentabilidad, los resultados muestran un coeficiente de rentabilidad de 84,14 para el caso de Sucre y un 80,4 para el caso de Córdoba (Tabla 2). Igual que en el caso anterior, favorable para el departamento de Sucre; aunque el ingreso neto es mayor para Córdoba en 6.440 .000 pesos colombianos, el costo de producir una tonelada de berenjena es mayor para este último departamento.

En cuanto a la eficiencia técnica del cultivo, se relacionó la diferencia entre el ingreso bruto y el costo directo con el área cultivada, como una manera de ver el aporte por área a la rentabilidad, y se obtuvo un coeficiente de $\$ 3.539 .000$ para el caso del departamento de Sucre y de $\$ 6.394 .000$ para el caso del departamento de Córdoba. Esto se explica por el hecho de que aunque los costos son mayores, también lo son los rendimientos para el departamento de Córdoba, y esto compensa en gran medida el costo adicional, permitiendo el retorno de los ingresos invertidos. Igualmente, lo que está mostrando es que hay una respuesta técnica a los recursos utilizados en el cultivo.

Por último, en lo relativo a la eficiencia económica, que relaciona el precio de venta con el costo unitario de producción con valores de 1,51 para el departamento de Sucre y 1,53 para el departamento de Córdoba, es importante anotar que estos valores guardan relación con la eficiencia técnica y con la rentabilidad total, y son favorables al departamento Córdoba, pues en los dos casos se superan por amplio margen los costos de producción con los rendimientos obtenidos. Sin embargo, los aumentos en los costos para el caso del departamento de Córdoba se ven compensados con los rendimientos.

\section{A. Márgenes de comercialización}

Con la información obtenida de los diferentes actores: productor primario, que es el agricultor que está en la finca, el intermediario y el consumidor final, se calcularon los márgenes de comercialización: margen bruto de comercialización $(\mathrm{MBC})$, la participación directa del productor (PDP) y el margen neto de comercialización (MNC), como se explican a continuación.

\section{1) Margen bruto de comercialización (MBC):}

$$
\begin{gathered}
\text { MBC }=\frac{\text { PrecioConsumidor }- \text { Pr ecio Productor }}{\text { PrecioConsumidor }} * 100 \\
\text { MBC }=\frac{\$ 2.000-\$ 350}{\$ 2.000} * 100=82,5 \%
\end{gathered}
$$


Los precios están calculados en pesos colombianos, los precios al productor fueron obtenidos del promedio que le pagan al productor en la finca a $350 \$ / \mathrm{kg}^{-1} \mathrm{y}$ del promedio del precio al consumidor en los mercados de las principales ciudades de la región Caribe. En este sentido, el margen bruto de comercialización se define como la proporción que paga el consumidor por cubrir los gastos y utilidades del vendedor final o la intermediación total, es decir que por cada $\$ 1$ que paga el consumidor, $\$ 0,82$ son captados por la intermediación. Es una suma muy alta, si se tiene en cuenta que la intermediación se lleva un porcentaje muy alto de esta diferencia.

Para el caso del intermediario, se calculó usando la misma fórmula, como se indica a continuación:

MBC $=\frac{\$ 2.000-\$ 1.300}{\$ 2.000} * 100=53,8 \%$

La proporción que paga el consumidor por cubrir los gastos y utilidades del intermediario, es decir, que por cada $\$ 1$ que paga el consumidor, $\$ 0,53,8$ son captados por el segundo eslabón de la cadena, o sea el intermediario que le compra en la finca al productor.

\section{2) Participación directa del productor (PDP):}

$P D P=100-M B C=100-82,5=17.5 \%$

De acuerdo con este indicador, el productor recibe $\$ 0,17$ por cada $\$ 1$ que paga el consumidor, es decir que el $17 \%$ de lo que paga el consumidor es la cantidad que realmente recibe el agricultor.

Con la misma metodología se calculó la participación directa del intermediario, que dio un valor de $46.2 \%$, lo que significa que el intermediario recibe $0,46,2$ pesos por cada unidad monetaria que paga el consumidor.

3) Margen neto de comercialización (MNC):

MNC $=\frac{\text { Pconsu }- \text { Pprod }- \text { CostosdeMercadeo }}{\text { Pr ecioConsumidor }}=\frac{(\$ 2.000-\$ 350)-\$ 200}{\$ 2.000} * 100$

MNC $=72.5 \%$

Para el cálculo se asumió que el costo del mercadeo es de 200 pesos colombianos por kilo vendido, que corresponde al transporte de un kilo vendido. El resultado muestra que por cada $\$ 1$ que paga el consumidor, $\$ 0,72$ son utilidades que se distribuyen en la cadena de intermediación. Lo anterior permite ver que el margen de comercialización es muy alto, por cuanto la proporción del pago que hace el consumidor por el producto es del $17.5 \%$ del valor que paga y, por otra 
parte, la intermediación se lleva un $72.5 \%$ del precio pagado por el consumidor, y el intermediario es el que menos arriesga en el proceso. Estudios similares se encontraron en sistemas de producción agrícola, específicamente en el sistema de producción de yuca seca para alimentos balanceados. Martínez y Esquivia (2007) también calcularon los mismos indicadores y obtuvieron para el margen bruto el 27 $\%$ y para la participación directa del productor el $73 \%$. Los resultados, en el caso de la habichuela larga, coinciden en el hecho de que el margen bruto de comercialización es del $25 \%$ y la participación directa del productor es del $50 \%$.

\section{Conclusiones}

El manejo del sistema de producción incide en los resultados finales reflejados en los indicadores de retorno; como se pudo evidenciar, fue el mismo sistema de producción, pero en dos ambientes diferentes. La participación de la mano de obra y la menor participación del factor capital hicieron que fuera menor el costo de producción para el caso de la región de Sabanas; y para el caso del Valle del Sinú, aunque siendo más intensivo en el uso del capital, se vio compensado con un mayor ingreso neto, por cuanto los rendimientos también fueron mayores.

La composición de los costos de producción incide en las respuestas en los indicadores de retorno a la inversión. Una mayor participación en las labores, y en especial en la mano de obra, afecta positivamente los costos de producción. En este sentido, la proporción en participación de la mano de obra en los costos de producción es mayor en el caso del departamento de Sucre, con el $75 \%$ del total de los costos, lo que hace que el costo total sea más bajo para esta región con relación al departamento de Córdoba, lo cual permite también apreciar la poca dependencia de los insumos comprados en el mercado y por eso la producción se hace a un costo más bajo.

El análisis de los márgenes de comercialización pone al descubierto la poca integración de la cadena productiva y la desventaja del productor frente a los demás eslabones, pues, aunque es este el que más arriesga, solo le corresponde un $17.5 \%$ de lo que paga el consumidor, lo que muestra una inequidad en las transacciones.

\section{AGRADECIMIENTOS}

A los agricultores de la región Caribe, por su tiempo en el suministro de la información sobre tecnología local de producción. Al Ministerio de Agricultura y Desarrollo Rural (MADR) (C09495), por la financiación del proyecto "Análisis socioeconómico, tecnológico y de mercado de las principales hortalizas cultivadas en la Región Caribe de Colombia". A la Corporación Colombiana de Investigación Agropecuaria AGROSAVIA, por su apoyo en la gestión logística durante la vida del proyecto. 
Análisis económico de la producción de berenjena (Solanum melongena L.) en dos zonas productoras del Caribe colombiano: Sabanas de Sucre y Valle del Sinú en Córdoba

\section{REFERENCIAS}

Agreda, V., Quijandría, B., \& Ruiz, M. E. (1991). Metodología para el análisis económico: Aspectos metodológicos del análisis social en el enfoque de sistemas de producción: memorias. En Reunión de Trabajo Aspectos Metodológicos del Análisis Social en el Enfoque de Sistemas de Producción. Cajamarca (Perú), 27-31, 1990.

Agronet. (2018a). Estadísticas comparativo de área, producción y rendimiento de participación departamental por cultivo 2018. Recuperado de: https://www.agronet.gov.co/estadistica/Paginas/home.aspx?.cod=3.

Agronet (2018b). Área cosechada, producción y rendimiento de Berenjena 2018. Recuperado de http://www.agronet.gov.co.

Alam, M. N., Uddin, M. T., Moniruzzaman, M., Nazia, T., \& Haque, M. M. (2016). Socio-Economic Characteristics of the Tomato Farmers in Selected Areas of Chapainawabganj District. Fundamental and Applied Agriculture, 1(2), 101-105.

Araméndiz, T. H., Cardona, A. C., Jarma, O. A., \& Espitia, C. M. (2008). El cultivo de berenjena (Solanum melongena L). Bogotá: Produmedios.

Arvizu, B. E., Mayett, M. Y., Martínez, F. J., Olivares, B. E., \& Flórez, M. L. (2015). Análisis de producción y comercialización hortícola del estado de Puebla: un enfoque de cadena de valor. Revista Mexicana de Ciencias Agrícolas, 6(4), 779-792. https://doi.org/10.29312/remexca.v6i4.618.

CIMMYT. (1988). La formulación de recomendaciones a partir de datos agronómicos: un manual metodológico de evaluación económica. México D.F.: CIMMYT. Recuperado de https://repository.cimmyt.org/xmlui/bitstream/handle/10883/1063/9031.pdf.

Escobar, G., \& Berdegué, J. (1990). Tipificación de sistemas de producción agrícola. Santiago de Chile: Red Internacional de Metodología de Investigación de Sistemas de Producción (RIMISP).

Espinosa, P., Játiva, P., \& Suárez, G. (1990). Caracterización de los sistemas de producción agrícola de productores de maíz de la provincia de Bolívar en Ecuador. En G. Escobar \& J. Berdegué (ed.), Tipificación de sistemas de producción agrícola (pp. 157-166). Santiago de Chile. Recuperado de https://idl-bnc-idrc.dspacedirect.org/bitstream/handle/10625/3969/49675.pdf? sequence=1.

Espinosa, V. E., Rivera, G., \& García L. A. (2008). Los canales y márgenes de comercialización de la leche cruda producida en sistema familiar (estudio de caso). Veterinaria México, 39(1), 1-16.

Forero, J., Garay, L. J., Barberi, F., Ramírez, C., Suárez, D. M., \& Gómez, R. (2013). Reflexiones sobre la ruralidad y el territorio en Colombia. Problemáticas y retos actuales. En L. J. Garay et al., La eficiencia económica de los grandes, medianos y pequeños productores agrícolas colombianos (pp. 69-111). Bogotá: Oxfam.

Hernández, L., Cardona, A., C., Vergara, C. C., Araméndiz, T.H., \& Velásquez-Arteaga, R. (2015). Efecto de coberturas y micorrizas nativas sobre el cultivo de berenjena (Solanum melongena L.). Agron., 23(1), 719.

Krugman, P., \& Wells, R. (2006). Introducción a la microeconomía. Barcelona: Reverté.

Martínez, A., \& Esquivia, M. (2007). La competitividad de la yuca seca en la cadena avícola porcícola en Colombia. Comercio Exterior, 57(1), 20-23.

Martínez, A. M. (2013). Caracterización socioeconómica de los sistemas de producción de la región de La Mojana en el Caribe de Colombia. Corpoica Ciencia y Tecnología Agropecuaria, 14(2), 165-185. https://doi.org/10.21930/rcta.vol14 num2 art:406.

Pabón, M. (2016). Caracterización socioeconómica y productiva del cultivo de cacao en el departamento de Santander (Colombia). Revista Mexicana de Agronegocios, 38, 238-294.

Ríos, G., Romero, M., Botero, M. J., Franco, G., Pérez, J. C., Morales, J. E., \& Echeverry, D. I. (2004). Zonificación, caracterización y tipificación de los sistemas de producción de lulo (Solanum quitoense

Ciencia y Agricultura (Cien. Agri.) Vol. 16 (3). L-ISSN 0122-8420. elSSN 2539-0899.

Septiembre-Diciembre 2019, pp. 17-34. Tunja (Boyacá) - Colombia. DOI:

https://doi.org/10.19053/01228420.v16.n3.2019.9514 
Antonio María Martínez-Reina; Lilibet Tordecilla-Zumaqué; Liliana María Grandett-Martínez; María del Valle Rodríguez-Pinto; Carina Cecilia Cordero-Cordero; Alfonso Rafael Orozco-Guerrero; Gabriel Ernesto SilvaAcosta; Jorge Luis Romero-Ferrer; Ender Manuel Correa-Álvarez

Lam) en el Eje Cafetero. Corpoica Ciencia y Tecnología Agropecuaria, 5(1), 22-30. https://doi.org/10.21930/rcta.vol5 num1 art:21.

Rodríguez, J. (2005). Métodos de muestreos. Madrid: Centro de Investigaciones Sociológicas.

Tapia. J., Cadena, J., Correa, E., Jiménez, N., Rodríguez, M., Tamayo, P., \& Arias, H. (2015). Modelo tecnológico del cultivo de berenjena para la región Caribe. Mosquera, Colombia: Corpoica. Recuperado de https://repository.agrosavia.co/bitstream/handle/20.500.12324/13764/75843 65796.pdf?sequence=1\&isAll owed $=\mathbf{y}$.

Torres, E., Quisphe, D., Sánchez, A., Bermeo, M., González, B., Torres, A., \& Chong, H. (2013). Caracterización de la producción de frijol en la provincia de Cotopaxi Ecuador: caso Comuna Panyatug. Revista Ciencia y Tecnología, 6(1), 23-31.

Vallejos, J. (2012). Línea de base para el proyecto de desarrollo de la producción del cultivo de papa en el distrito de Tayabamba provincia de Pataz, departamento de la Libertad, Perú. Documento de trabajo. Recuperado de http://www.agrolalibertad.gob.pe/sites/default/files/linea\%20de\%20base\%20para\%20productores\%20de\% 20papa \%20del\%20distrito\%20de\%20tayabamba\%20departamento\%20la\%20libertad.pdf. 